Special Issue of the 8th International Advances in Applied Physics and Materials Science Congress (APMAS 2018)

\title{
Mayan Inspired Nanocomposite Materials: an Overview
}

\author{
R. Giustetto ${ }^{a, b, c, *}$ \\ ${ }^{a}$ Department of Earth Sciences, University of Turin, via Valperga Caluso 35, 10125 Torino, Italy \\ ${ }^{b}$ NIS - Nanostructured Interfaces and Surfaces Centre, via Quarello 15/A, 10135 Torino, Italy \\ ${ }^{c}$ INFN - National Institute of Nuclear Physics, via Giuria 5, 10125 Torino, Italy
}

\begin{abstract}
Maya Blue is a famous pigment used by ancient Mayas, renowned for its stability, formed after a heatinginduced encapsulation and bonding of the indigo dye inside the nano-tunnels crossing the structure of the clay mineral palygorskite. Such a discovery led to an inspiration: if Mayas made it blue, someone else could have made it any other colour! Some red dyes were thus complexed with palygorskite so to check, using a multi-analytical approach (including molecular mechanics, UV-Vis, FT-IR and SER spectroscopies and TGA-GC-MS), whether the ensuing compounds might have shown physical/chemical features similar to their notorious 'blue brother'. Two of the tested dyes gave notable results. When alizarin is grafted on palygorskite, a composite is formed that changes colour (orange-to-purple) after $\mathrm{pH}$ variations. Methyl red forms instead a stable red/purple complex with palygorskite, suffering no colour change despite severe acid/alkali attacks. Surface interactions form between alizarin and palygorskite, thus allowing the dye to maintain its sensitivity (and colour change) with pH. Conversely, methyl red can diffuse and bind in the clay nano-pores, forming supramolecular host/guest interactions responsible for the composite stability.
\end{abstract}

DOI: 10.12693 /APhysPolA.135.1123

PACS/topics: Maya Blue, palygorskite, methyl red, alizarin, hybrid nanocomposite

\section{Introduction}

The exceptional features of innovative nanocomposite materials are presented here, which have been synthesized drawing an inspiration from the past. In Precolumbian America, back in the VI century AD, the ancient Mayas created the so-called Maya Blue pigment an ancestor of modern nano-composite materials, used for mural paintings and decorations. When re-discovered in modern times, Maya Blue grabbed the interest of the scientific community due to its astounding stability. The pigment can resist the attack of acids, alkalis and solvents, without losing its structural features nor showing any colour fading. The secrets of Maya Blue invulnerability lasted until the rise of the new millennium. The pigment forms through a heating-induced encapsulation and bonding of the indigo dye inside the micropores of the clay mineral palygorskite, the structure of which is crossed by nano-tunnels filled by weakly bound (zeolitic) $\mathrm{H}_{2} \mathrm{O}$. Tightly bound structural $\mathrm{OH}_{2}$ completes instead the coordination of octahedral Mg. Heating causes loss of zeolitic $\mathrm{H}_{2} \mathrm{O}$, favouring dye diffusion inside the clay tunnels and formation of host/guest interactions, responsible for the composite stability [1, and references therein]. Since Mayas made it 'blue'... why not make it 'red', or 'green', or whatever! Basing on previous, pioneering attempts $[2,3]$, new hybrid composites were thus prepared by "marrying" palygorskite with some red dyes, accordingly to the recipe followed for Maya Blue, with the aim of creating a palette of environment-friendly pigments, with

*e-mail: roberto.giustetto@unito.it all possible hues (starting from the red one), granted by limited toxicity and low costs. Their physical-chemical properties are summarized in this brief overview.

\section{Experimental procedure}

Natural palygorskite, coming from Mexico (Chiapas State), was mixed with each of the selected red dyes - namely methyl red, alizarin, murexide and Sudan red, purchased from Sigma-Aldrich - in proper amounts $(2 \mathrm{wt} \%)$, crushed and heated $\left(160^{\circ} \mathrm{C}\right)$. Soxhlet extraction in apt solvents was applied to wash away the excess dye. Fixation of the red dyes on the host framework and their mutual interactions were tested with a multi-analytical approach, which includes molecular mechanics (atomistic lattice energy minimizations; Discover 2009.1 program as implemented in the Materials Studio 5.0 package of Accelrys Inc.) and spectroscopic methods, such as UV-visible (Varian Cary 5000 spectrophotometer), FT-IR (Bruker Vector 70, resolution: $2 \mathrm{~cm}^{-1}$; 64 scans/spectrum) and Surface-Enhanced Raman (Renishaw in-Via micro-Raman spectrometer). An in-line thermogravimetry-gas chromatography-mass spectrometry operating system (Perkin-Elmer, Spectrum 100, Clarus 500S gas chromatograph) was also used.

\section{Results and discussion}

Two out of the four tested dyes (alizarin and methyl red) maintained their red/purple hue after sorption on palygorskite. A drastic colour change was instead observed for murexide, which turned to green after heating, while Sudan red lost its colour after Soxhlet treatment. Further analyses were then performed only on those composites that evidenced successful complexation between the clay and the dye. 

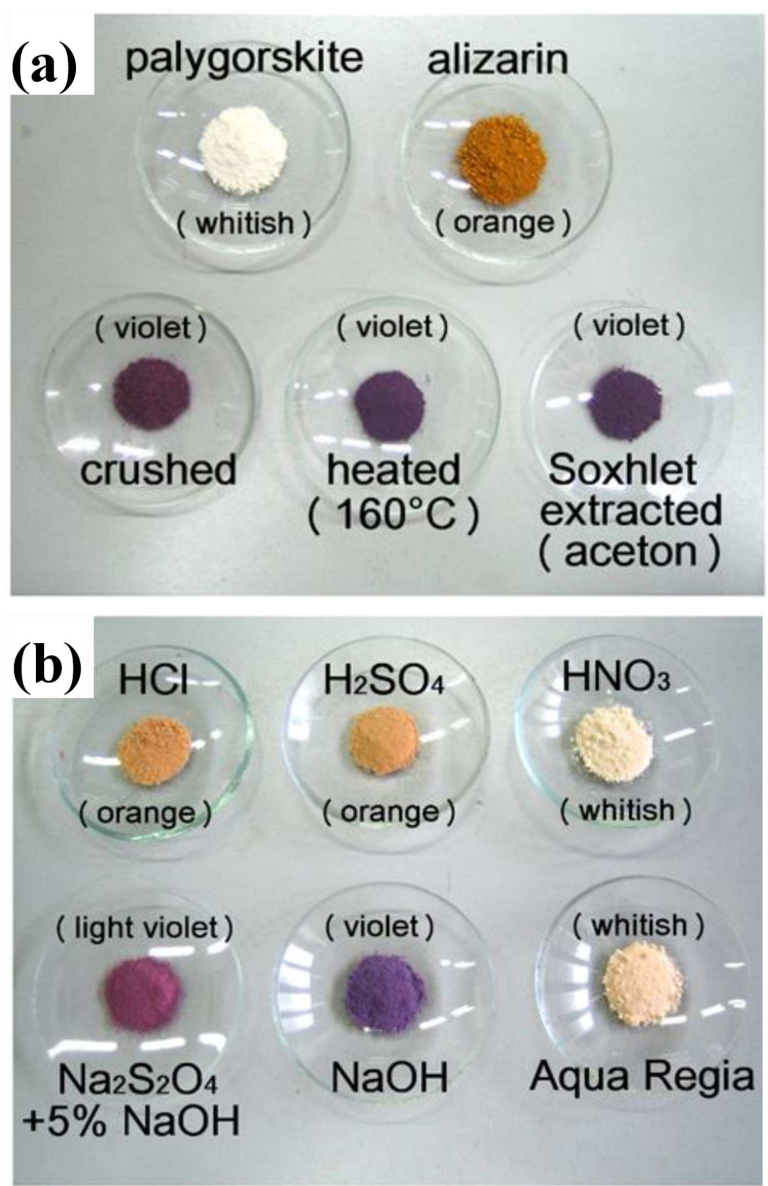

Fig. 1. The 'palygorskite + alizarin' composite: (a) steps of the synthesis, (b) after acid/alkali attacks (powder colours are indicated in brackets).

Pristine alizarin has a typical orange hue, but when crushed with palygorskite a purple composite is obtained, the color of which is maintained after heating and Soxhlet treatment (Fig. 1a). UV-visible diffuse reflectance spectroscopy, used to investigate this colour change, shows that in pure, solid-state alizarin the neutral form of the dye prevails (band at $442 \mathrm{~nm}$ ), which is consistent with its orange hue. When ground with palygorskite, however, the dye switches to its mono-anionic form (band red shifts at $515 \mathrm{~nm}$ ) well before heating, due to interaction and formation of specific host/guest bonds with the clay matrix.

FT-IR shows appearance in the composite spectrum of a band at $1524 \mathrm{~cm}^{-1}$, which finds no counterpart in the pure dye, accounting for the existence of surface interactions between the mono-anionic form of alizarin and the surface silanols of palygorskite and implying formation of a 'salt' structural model. Strong chemical attacks cause visible changes in the composite appearance (Fig. 1a, b): in acids and aqua regia, its hue turns towards yellowto-orange tones, whereas alkali attack causes no relevant colour change. This suggests that alizarin, when fixed on palygorskite, can exhibit different electronic configurations as a function of $\mathrm{pH}$ fluctuations, each state being
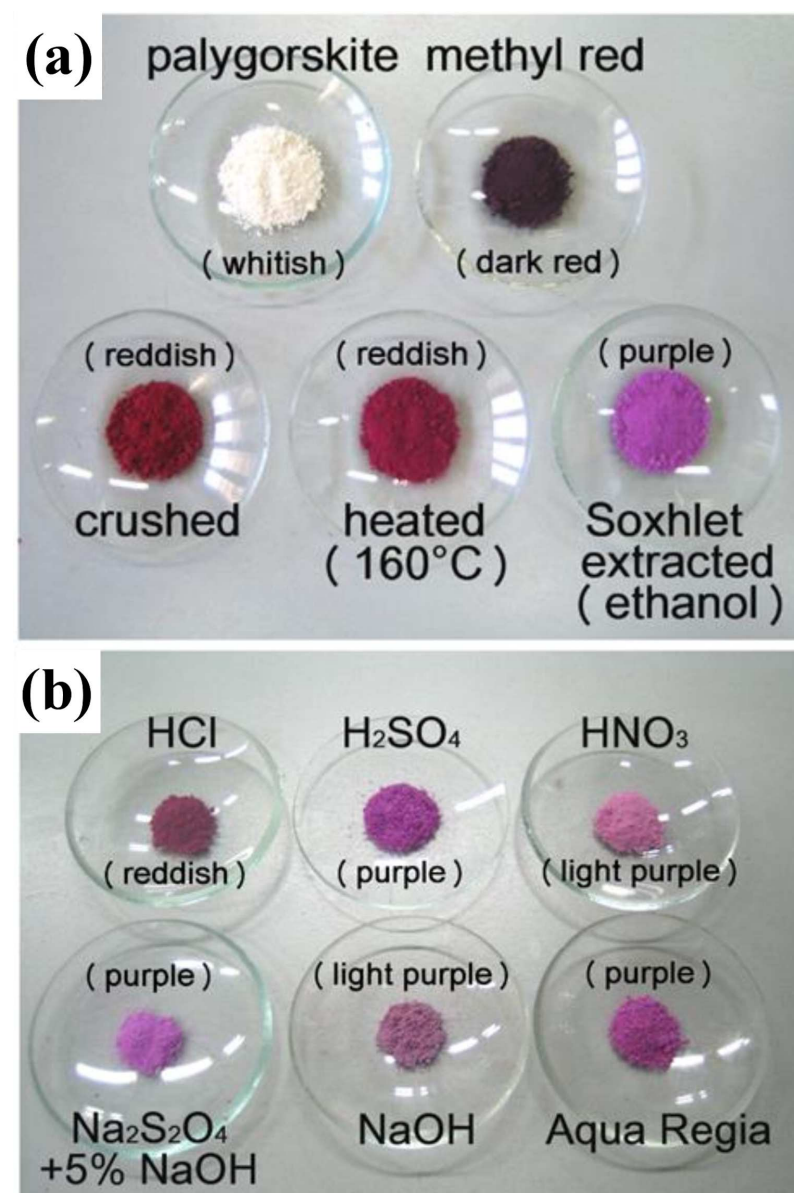

Fig. 2. The 'palygorskite + methyl red' composite: (a) steps of the synthesis, (b) after acid/alkali attacks (powder colours indicated in brackets).

associated to specific hues. Before crushing, alizarin molecules - still far from the clay surface - are in neutral state with an orange hue. Strong crushing causes an electrostatic interaction to occur between alizarin and the clay superficial $\mathrm{SiO}^{-}$oxyanions, switching the dye to its mono-anionic form and consequent colour change to purple. These superficial host/guest interactions allow alizarin to maintain its sensitivity to $\mathrm{pH}$. Though unfit to be used as a pigment (its colour changing with $\mathrm{pH}$ ), this 'palygorskite + alizarin' composite could thus represent a valid, solid-state $\mathrm{pH}$ sensor [4].

When palygorskite is ground and heated with methyl red - an azo dye used as a pH indicator - a brilliant and stable red/purple complex is obtained (Fig. 2a), which basically maintains its colour despite severe acid and/or alkali attacks (Fig. 2b). This hybrid composite can therefore be rightfully christened 'Maya Red' [4].

FTIR spectra, collected on this 'palygorskite + methyl red' composite in various synthesis steps and controlled $T$ /vacuum conditions, show existence of supramolecular host/guest interactions, as evidenced by the shift and intensity variations of certain dye modes before and after complexation with the clay (e.g., bands at 1277, 1312 and $\left.1398 \mathrm{~cm}^{-1}\right)$. Persistence at high $T$ of a doublet at 3690 
and $3714 \mathrm{~cm}^{-1}$ indicate an approaching of methyl red reactive groups $\left(\mathrm{CH}_{3}\right.$ or phenyl) to the hexagonal hole of the amphibole-like tetrahedrons chains of palygorskite, suggesting feasible dye encapsulation. TGA shows that sorption of methyl red affects the clay dehydration, varying the release of both zeolitic $\mathrm{H}_{2} \mathrm{O}$ (decreased weight loss at $120^{\circ} \mathrm{C}$, implying a smaller amount occupies the tunnels) and structural $\mathrm{OH}_{2}$ (additional event at $400^{\circ} \mathrm{C}$ ), which are expected to play a key role in forming mutual bonds between the host and the guest. Molecular mechanics confirmed that the secret of the "palygorskite + methyl red' composite stability is the fact that — as per its renowned 'blue brother' — the guest dye diffuses in the host pores and is stabilized by specific interactions, which show a typical evolution with $T$ rise. In particular, below $300^{\circ} \mathrm{C}, \mathrm{H}$-bonds form between the clay structural $\mathrm{OH}_{2}$ and the carboxyl group of methyl red; above that threshold, partial structural $\mathrm{OH}_{2}$ loss implies straight bonds to form between the dye $\mathrm{COOH}$ and the octahedral Mg-ions of palygorskite. Once the composite is cooled, though, these latter interactions tend to reverse to structural $\mathrm{OH}_{2}$-mediated $\mathrm{H}$-bonds, consistently with the host rehydration. At $T \geq 400{ }^{\circ} \mathrm{C}$ decay of the guest dye occurs, alongside a visible composite bleaching; since pure methyl red decomposes at $200{ }^{\circ} \mathrm{C}$, its thermal stability is thus virtually doubled by sheltering in the host framework. At $500^{\circ} \mathrm{C}$ ejection from the clay pores occurs, as decayed dye moieties (benzene and $\mathrm{Cl}$-benzene, the latter related to $\mathrm{HCl}$ addition during synthesis) are identified by gas chromatography (peaks at $5.4^{\prime}$ and $7.25^{\prime}$ ) and mass spectroscopy in the TGA outsourcing fumes [5]. SER spectra show that bands related to both the neutral and protonated dye species coexist in the tunnels, thus acknowledging that various methyl red isomers are hosted in palygorskite. Speciation of the dye is favoured by addition of $\mathrm{HCl}$ during synthesis and further dye protonation after encapsulation [6].

\section{Conclusion}

New 'Mayan-inspired' compounds can be prepared by 'marrying' palygorskite to other guest dyes, thus obtaining polyfunctional organic/inorganic hybrid composites useable in the materials science and cultural heritage fields. These composites might represent ecological surrogates to noxious compounds used nowadays. Further perspectives aim at synthesizing similar complexes with other hues, as well as testing the suitability of palygorskite as a carrier for new organic guests (e.g., active principles of drugs).

\section{Acknowledgments}

G. Ricchiardi, S. Bordiga, J.G. Vitillo, F. Turci and I. Corazzari are thanked for their contributions.

\section{References}

[1] M. Sánchez del Río, A. Doménech, M.T. DoménechCarbó, M.L. Vazquez de Agredos Pascual, M. Suárez, E. García Romero, The Maya Blue pigment, in: Developments in palygorskite-sepiolite research, a new outlook on these nanomaterials, Eds. E. Galan, A. Singer, Elsevier, Oxford 2011, p. 453.

[2] D. Reinen, P. Köhl, C. Muller, Zeitschrift Anorganische Allgemeine Chem. 630, 97 (2004).

[3] F.S. Manciu, A. Ramirez, W. Durrer, J. Govani, R.R. Chianelli, J. Raman Spectrosc. 39, 1257 (2008).

[4] R. Giustetto, O. Wahyudi, Micr. Mesop. Mater. 142, 221 (2011).

[5] R. Giustetto, J.G. Vitillo, I. Corazzari, F. Turci, J. Phys. Chem. C 118, 19322 (2014).

[6] R. Giustetto, A. Idone, E. Diana, J. Raman Spectrosc. 48, 507 (2017) 